\title{
Electronic resonance states in metallic nanowires during the breaking process simulated with the ultimate jellium model
}

\author{
E. Ogando, ${ }^{1, *}$ T. Torsti, ${ }^{2,3}$ N. Zabala, ${ }^{1,4}$ and M. J. Puska ${ }^{2}$ \\ ${ }^{1}$ Elektrika eta Elektronika Saila, UPV-EHU 644 Posta Kutxa, 48080 Bilbo, Spain \\ ${ }^{2}$ Laboratory of Physics, Helsinki University of Technology, P.O. Box 1100, FIN-02015 HUT, Finland \\ ${ }^{3}$ CSC-Scientific Computing Ltd., P.O. Box 405, FIN-02101 Espoo, Finland \\ ${ }^{4}$ Donostia International Physics Center (DIPC), 1072 Posta Kutxa, 20080 Donostia, Spain
}

(Received 03 September 2002; published 28 February 2003)

\begin{abstract}
We investigate the elongation and breaking process of metallic nanowires using the ultimate jellium model in self-consistent density-functional calculations of the electronic structure. In this model the positive background charge deforms to follow the electron density and the energy minimization determines the shape of the system. However, we restrict the shape of the wires by assuming rotational invariance about the wire axis. First, we study the stability of infinite wires and show that the quantum-mechanical shell structure stabilizes the uniform cylindrical geometry at the given magic radii. Next, we focus on finite nanowires supported by leads modeled by freezing the shape of a uniform wire outside the constriction volume. We calculate the conductance during the elongation process using the adiabatic approximation and the WKB transmission formula. We also observe the correlated oscillations of the elongation force. In different stages of the elongation process two kinds of electronic structures appear: one with extended states throughout the wire and one with an atom-cluster-like unit in the constriction and with well-localized states. We discuss the origin of these structures.
\end{abstract}

DOI: 10.1103/PhysRevB.67.075417

PACS number(s): 73.21.Hb, 73.40.Jn, 73.63.Nm

\section{INTRODUCTION}

The miniaturization of the electronic components is of great importance in the development and improvement of new devices for applications in a wide number of fields. Although the laws of nature are the same for macroscopic and mesoscopic systems, the miniaturization process is achieving the limit where the quantum behavior of matter starts to play an important role.

If the size of the system under consideration is only a few nanometers, the atomic character of matter emerges and it cannot be considered as a continuum. The regime of quantum behavior is reached also if one of the spatial dimensions of the system is reduced down to the Fermi wavelength of the conducting electrons. Then, the confinement splits the continuous electronic band in this direction into a set of discrete energy levels. In both cases, the behavior of the system changes from what is expected from the macroscopic case. In metallic nanowires the Fermi wavelength is of the same order of magnitude as the atomic distance, and both atomic and electronic discrete character compete and/or couple, determining the properties of nanowires.

There are many experimental and theoretical works that have gone deep into the understanding of the main features of nanowires. Experimental studies have focused on the investigation of the mechanical and electronic properties, such as force, atomic structures, and conductance, pointing out the close relation between them. Among the experimental setups, we want to emphasize the role of the scanning tunneling microscope $^{1-3}$ (STM) and the mechanically controllable break-junction (MCBJ) techniques. ${ }^{4-6}$ In both techniques metallic nanowires are produced by putting two protrusions in contact and then pulling them away from each other over atomic distances. In this process, a nanowire is produced which upon pulling is elongated and narrowed until it eventually breaks. These methods have allowed the study of transport properties and stability of nanowires.

The MCBJ techniques have demonstrated the existence of electronic and atomic shell structures ${ }^{4-6}$ analogous to those found in atomic clusters. ${ }^{7,8}$ In these experiments the conductance has been studied by building histograms of the conductance during the breaking process. The results show that there are conductance values that are much more probable than others. Due to the relation between the conductance and the radius at the narrowest part of the nanowire, which means that there are magic radii with enhanced stability while other radii are less stable, and therefore they appear less frequently in the conductance histograms. The atomic structures of nanowires in the last steps before breaking have been also studied with these techniques., ${ }^{2,9-12}$

The experiments discussed above have been accompanied by supporting theoretical investigations that can be split in two groups. The first group includes classical and ab initio molecular-dynamics simulations, in which the atomic structure of nanowires is taken into account. These investigations have been successful in many aspects, e.g., showing the atomistic mechanisms of the narrowing process (appearance of dislocations, order-disorder stages, etc.) and their link with other measurable quantities such as the elongation force or the conductance. ${ }^{13,14}$ Moreover, from the viewpoint of the present work, we notice the predictions of special atomic arrangements in STM tips and nanowire necks. ${ }^{13,15-19}$ The second group of models is more related to the properties due to the confinement of electrons in reduced dimensions, and ignores the atomistic structure of matter. In these calculations, analytic approximations as well as self-consistent electronic-structure models have been used, mainly within 
the jellium framework. The results obtained with these methods are also enlightening, explaining the cohesive and electronic transport properties of nanowires, especially in the case of alkali metals with strong free-electron character. ${ }^{20-24}$

The aim of this paper is to simulate the breaking of nanowires. For this purpose we choose the jellium model and the self-consistent electronic-structure calculations within the density-functional theory. In spite of their simplicity, jellium models have provided a simple and transparent way to understand the physics of metallic nanowires. More specifically, we use the ultimate jellium (UJ) model. This model was first proposed by Manninen ${ }^{25}$ to investigate the structures of alkali-metal clusters. It has been used for the same purpose also in later studies. ${ }^{26,27}$ To our knowledge the present work is the first time the UJ model is used to simulate the nanowire breaking. In practice, we solve the ensuing Kohn-Sham equations in a real-space point grid using the powerful Rayleigh quotient multigrid ${ }^{28,29}$ (RQMG) method implemented in the program package MIKA (Multigrid Instead of K-spAce). ${ }^{30}$

Within the UJ approach, the background positive charge density is fully relaxed in shape and density so that it equals at every point with the electron density. One can think that this freedom of the positive background charge mimics the efficient rearrangement and diffusion of ions at temperatures close to the melting point at which the shell- and supershellstructure studies by the MCBJ techniques have been performed for alkali metals. ${ }^{4,5}$ In principle, there is no restriction for the geometry of the constriction. This is in contrast with the previous jellium calculations that introduced ad hoc shapes for the nanowire. In our model the electrons themselves acquire self-consistently the shape, which minimizes the Kohn-Sham energy functional, and carry along the positive background charge. However, in order to reduce computational demands and to highlight the important phenomena from the complexity of possible solutions, we restrict the shapes of nanowires to the axial symmetry, i.e., rotational invariance with respect to an axis.

One of our main results is that in the narrowest part of the nanowire, electronic cluster derived structures ${ }^{13,19}$ (CDS's) appear. This tendency of electrons to form embedded clusters in the jellium constrictions is analogous to the preferred clusterlike arrangements of atoms in contacts, described by the first-principles atomistic calculations by Barnett and Landman. ${ }^{13,19}$ CDS's have later been reported also by other authors. ${ }^{15}$ The main difference is that in our jellium model the atomistic character of the previous works is lost and the electrons alone are responsible for the phenomenon. The single-electron states provided by the jellium model can be studied in order to gain insight into the localization effects associated with the CDS. The conductance of the constriction can be estimated either by counting the bands crossing the Fermi level or by using the WKB formula.

The rest of the paper is organized as follows: in Sec. II, we describe the practical features of the UJ model and the RQMG method to calculate the electronic structure during the elongation process. In Sec. III, we discuss the results for the electronic properties. As a starting point, we consider the results for infinite wires. Then we focus our attention on the breaking process of a finite cylindrical UJ nanowire supported by leads. Section IV contains the conclusions.

\section{THEORY}

\section{A. Jellium models}

The jellium model has been widely used in self-consistent electronic-structure calculations of nanostructures. It simplifies the problem by replacing the ions by a uniform rigid positive-charge-density background that globally neutralizes the electron negative charge. The effective potential of the Kohn-Sham ${ }^{31}$ equations is written as (atomic Hartree units are used throughout this paper in the equations)

$$
V_{\mathrm{eff}}(\mathbf{r})=\int \frac{n_{-}\left(\mathbf{r}^{\prime}\right)-n_{+}\left(\mathbf{r}^{\prime}\right)}{\left|\mathbf{r}-\mathbf{r}^{\prime}\right|} d r^{\prime}+V_{\mathrm{xc}}\left[n_{-}(\mathbf{r})\right],
$$

where the first term on the right-hand side includes the electron-electron and electron-positive background Coulomb interactions, and the second term gives the exchangecorrelation potential within the local-density approximation. ${ }^{32,33}$

Different types of jellium approaches have been introduced. The simple jellium (SJ) model has the problem that there is only one equilibrium charge density, at $r_{s} \approx 4.18 a_{0}$ $\left[n_{-}=3 /\left(4 \pi r_{s}^{3}\right)\right]$, corresponding approximately to the average conduction-electron density in Na. This means that for $r_{s}$ values lower (higher) than $\sim 4.18 a_{0}$, the jellium system tends to expand (compress). In the SJ model, the electron density has the same mean value as the positive background due to the electrostatic forces. The SJ model gives incorrect values for properties such as the cohesive energy, surface energy, and bulk modulus, due to the trend of the system to compress or expand. To improve the results, corrections can be added to the SJ model, ${ }^{34}$ e.g., using the so-called stabilized jellium model introduced by Perdew co-workers ${ }^{35}$ and Shore Rose. ${ }^{36}$

In this work, we use the UJ model, the philosophy of which differs from the stabilized jellium model in which it does not try to correct the above-mentioned deficiencies of the SJ model. The peculiarity of the UJ model is that the positive-charge background is allowed to relax. The UJ model represents the ultimate limit in which the positive background is completely deformed to have the same density as the electrons locally at every point. In this way, the Coulomb term in the potential always vanishes, and in Eq. (1) only the exchange-correlation term survives. The total energy is then minimized in the interplay between the exchange-correlation and the kinetic energies.

One limitation of the UJ model is that, as in the SJ model, there is only one equilibrium charge density at $r_{s} \approx 4.18 a_{0}$. But, the absence of electrostatic potential disables the mechanism to keep the electrons at a given density, and inside the UJ the mean electron density becomes equal to the equilibrium density. Another property of the UJ model, derived also from the absence of electrostatic potential, is that the shape of the electron density is to a large extent uncontrollable, and it evolves until the ground state is achieved. This property has been used to study the most favorable 
shapes of simple-metal-atom clusters. ${ }^{25-27,37}$ In the present work, however, we have to deal with open systems and we have to impose certain controlling restrictions in order to model the pulling of the nanowires. The description of the solutions to these requirements is postponed to Sec. III C.

\section{B. Numerical methods}

In Sec. III A, infinite uniform cylindrical wires are studied. Since these systems are translationally invariant along the wire axis, the relaxation of the positive background charge and electron density is limited in the radial direction. Consequently, it is necessary to solve numerically only the radial part of the Schrödinger equation (see Zabala et $_{\text {al. }}{ }^{22}$ for technical details).

For the systems studied in Secs. III B and III C, however, the translational invariance is not required. But, in addition to the rotational invariance, periodicity in the axial direction is assumed with unit-cell length $L_{\text {cell }}$. Thus, the wave functions $\psi$ are indexed by the quantum numbers $m, n$, and $k_{z}$. Here, $m$ is the angular momentum quantum number and $k_{z}$ is the Bloch wave vector along the wire axis. With $m$ and $k_{z}$ given, $n$ enumerates the orthogonal states in the order of increasing energy eigenvalue. The UJ system is solved by finding the self-consistent solution to the following set of equations:

$$
\begin{gathered}
\psi_{m k_{z} n}(r, z, \phi)=e^{i m \phi} U_{m k_{z} n}(r, z), \\
U_{m k_{z} n}\left(r, z+L_{\mathrm{cell}}\right)=e^{i k_{z} L_{\mathrm{cell}} U_{m k_{z} n}(r, z) .} \\
-\frac{1}{2}\left(\frac{1}{r} \frac{\partial}{\partial r}+\frac{\partial^{2}}{\partial r^{2}}-\frac{m^{2}}{r^{2}} \frac{\partial^{2}}{\partial z^{2}}+2 V_{\mathrm{eff}}(r, z)\right) U_{m k_{z} n}(r, z) \\
=\varepsilon_{m k_{z} n} U_{m k_{z} n}(r, z) . \\
n(\mathbf{r})=2 \sum_{m k_{z} n}\left(2-\delta_{0 m}\right) f_{m k_{z} n}\left|U_{m k_{z} n}(\mathbf{r})\right|^{2}, \\
V_{\mathrm{eff}}(r, z)=V_{\mathrm{xc}}(r, z)=\frac{\delta E_{\mathrm{xc}}[n(r, z)]}{\delta n(r, z)} .
\end{gathered}
$$

The effective potential $V_{\text {eff }}(r, z)$ equals the exchangecorrelation potential $V_{\mathrm{xc}}(r, z)$. The electron density $n(r, z)$ is obtained by summing single-electron densities with the occupation numbers $f_{m k_{z} n}$. The degeneracies of the states are taken into account by the factor $2\left(2-\delta_{0 m}\right)$, and the occupation numbers $f_{m k_{z}{ }^{n}}$ obey the Fermi-Dirac statistics with the Fermi level $\left(E_{F}\right)$ so that the system is neutral. A finite temperature of $1200 \mathrm{~K}$ is used to stabilize the solution of the set of equations.

The Schrödinger equation (4) is discretized on a regular two-dimensional $(r, z)$ point mesh. We use standard fourthorder central-difference discretizations for the first and the second derivatives. The grid is surrounded by a frame with the thickness of two grid points. These ghost points are necessary for the evaluation of the derivatives near the edges of the computation volume. The wave functions are required to vanish at the ghost points corresponding to the radial surface of the cylindrical computation volume, whereas at the axis the values at ghost points can be evaluated by noting that $U(-r, z)=(-1)^{m} U(r, z)$. The Bloch condition [Eq. (3)] gives the recipe for obtaining the values at the ghost points of the periodic boundary.

The problem with standard real-space relaxation methods for Eq. (4) is the so-called critical slowing-down phenomenon resulting from the fact that at a time they use information from a rather localized region of space. As a result of the locality, the high-frequency error, corresponding to the length scale of the grid spacing, is reduced very rapidly in the relaxation. However, once the high-frequency error has been effectively removed, the very slow convergence of the low-frequency components dominates the overall error reduction rate, i.e., critical slowing down occurs. Multigrid methods avoid this problem by treating the low-frequency components of the error on coarser grids, where their wavelength is comparable to the grid spacing.

Applying the multigrid methods to the Schrödinger equation is a fairly complicated task because one has to solve both the eigenvalue and the wave function simultaneouslythis makes the problem nonlinear. Also, one has to solve several wave functions simultaneously, avoiding the bottleneck of orthogonalizations as well as possible. The standard methods based on the full-approximation-storage ${ }^{38}$ method require that the wave functions are well representable on the coarsest grid used, implying severe limitations on the acceleration obtained by the multigrid idea. We use the recently developed generalization of the RQMG method $^{28,29}$ as implemented in the MIKA package, ${ }^{30}$ which avoids the problems described above. In short, one applies the Gauss-Seidel method on the finest grid. On the coarser grids one applies coordinate relaxations on the functional

$$
\frac{\left\langle\psi_{n}|H| \psi_{n}\right\rangle}{\left\langle\psi_{n} \mid \psi_{n}\right\rangle}+\sum_{i=1}^{n-1} q_{i} \frac{\left.\left\langle\psi_{i} \mid \psi_{n}\right\rangle\right|^{2}}{\left\langle\psi_{i} \mid \psi_{i}\right\rangle\left\langle\psi_{n} \mid \psi_{n}\right\rangle}
$$

This functional, which is actually defined on the finest grid, is the sum of the Rayleigh quotient and a penalty functional, which is introduced to ensure the orthogonality. Moreover, the relaxations are performed simultaneously for all wave functions. See Heiskanen et l. $^{29}$ for a more thorough discussion of technical details.

The Kohn-Sham equations have to be solved selfconsistently. In other words, one has to iterate until the output potential $V_{\text {eff }}$ obtained from Eq. (6) equals the input potential $V_{\text {eff }}$ that is used in Eq. (4). In typical cases of electronic-structure calculations, to avoid divergence due to charge sloshing, one uses sophisticated strategies to construct the input potential for the next iteration as an optimized mixture of input and output potentials of previous iterations. ${ }^{39,40}$ In the UJ iterations, however, the output potential can be taken directly as the input potential of the next iteration resulting in a rapid convergence. This is because of the absense of the long-range Coulomb interaction, which is the cause of the charge sloshing phenomenon. 


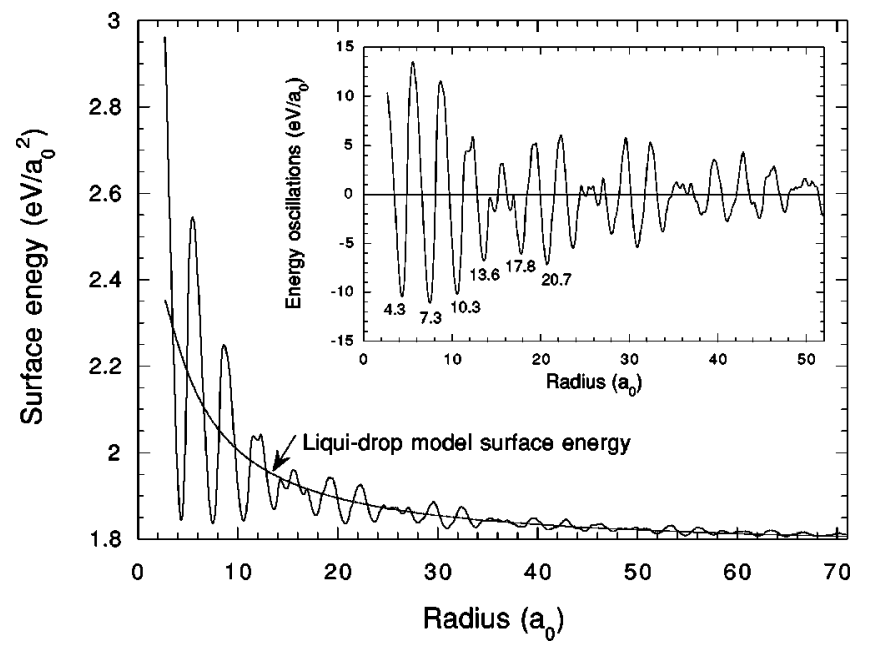

FIG. 1. Surface energy of infinite uniform cylindrical UJ nanowires as a function of the nominal wire radius (see text). In the inset the energy oscillations are shown and the first magic radii are marked.

\section{RESULTS AND DISCUSSION}

\section{A. Infinite uniform cylindrical wires}

The main results of this paper concerning the nanowire breaking process are discussed in Secs. III B and III C. As a preliminary work, and in order to gain insight into the UJ model in comparison with the stabilized jellium model, we study the stability of infinite uniform cylindrical UJ wires.

We calculate the surface energy of the nanowires and the oscillations in the energy per unit length as it was made in our previous work describing $\mathrm{Al}, \mathrm{Na}$, and $\mathrm{Cs}$ nanowires within the stabilized jellium model. ${ }^{20,21}$ The results are shown in Fig. 1 as a function of the wire radius $R$. Here the radius is defined as the radius of the positive background charge in the SJ system with $r_{s}=4.18 a_{0}$ and the same amount of charge per unit length. In order to separate the energy oscillation from the average behavior, the so-called liquid-drop model ${ }^{41}$ is used. In this model the energy of the jellium system can be written as the sum of two terms-one proportional to the volume and the other proportional to the surface area. For the first term, the energy/volume ratio corresponds naturally to the homogeneous electron gas ${ }^{42}$ with $r_{s}=4.18 a_{0}$. This view has been tested in clusters and nanowires ${ }^{20-22}$ and it describes correctly the mean energy, i.e., without the characteristic oscillations due to the quantum confinement. We fit the self-consistently calculated total energy per unit length to a liquid-drop-model-type function. Then, subtracting this smooth energy function from the total energy we get the pure energy oscillations, which are shown in the inset of Fig. 1. Note that there are radii for which the energy is at minimum. They correspond to wires that are more stable than wires with slightly different radii and higher energies. The first magic radii are $R=4.3 a_{0}, 7.3 a_{0}, 10.3 a_{0}$, $13.6 a_{0}, 17.8 a_{0}$, and $20.7 a_{0}$. We use these radii for the initial uniform wires in the nanowire breaking simulation in Sec. III C. The shell and super shell structures studied in previous calculations $^{20,21}$ are also quite clear. In comparison with the energy oscillations of $\mathrm{Na}$, we observe that the beat positions are shifted to higher radii. The reason is that the UJ potential is softer at the surface than the stabilized jellium potential for $\mathrm{Na}{ }^{20}$

\section{B. Periodic systems}

Now we change the scheme and allow the wire to deform also in the axial direction. However, we impose periodic boundary conditions with the unit-cell length $L_{\text {cell }}$ along the wire axis. $L_{\text {cell }}$ is thus the maximum perturbation wavelength in our calculation. From the liquid-drop model point of view, neglecting the small contribution of the curvature energy, the liquid wire attempts to achieve the shape that minimizes the surface, and thereby the total energy. Under this assumption an infinite periodic liquid wire is a uniform cylinder for lengths $L_{\text {cell }}<4.5 R$. For $L_{\text {cell }}>4.5 R$, it deforms trying to achieve the energetically most favorable state, an infinite chain of spheres. However, Kassubek et al. ${ }^{43}$ showed using a semiclassical model and perturbation theory that due to the discreteness of electronic structure, the wires with magic radii remain uniform also at large $L_{\text {cell }} / R$ ratios. With this result they argued that in the narrowing process of an infinite wire, when the radius is crossing an unstable zone before the next stable radius is achieved, the wire would spontaneously deform acquiring a wavy or deformed shape. We corroborate these results nonperturbatively using the UJ model as follows.

We choose a certain radius $R$ and solve for the UJ electronic (and positive-charge density) structures imposing increasingly longer supercell lengths $L_{\text {cell }}$ by increasing the number of electrons in the cell. Thereby we determine the critical supercell length (the wavelength of a perturbation) at which the wire starts deforming. For magic wires we find no wavy solutions, the wires remain uniform. For example, for $R=7.3 a_{0}$ the wire is still uniform at $L_{\text {cell }} / R \approx 36$. The wires corresponding to the radii at the maxima of the energy oscillations in Fig. 1 are the most unstable ones. These wires are uniform up to a critical value of $L_{\text {cell }}$, but above it they spontaneously deform to a wavy or nonuniform density profile along the wire axis. As an example, Fig. 2 shows the behavior of a wire with radius $R=5.5 a_{0}$ when the number of electrons in the unit cell is $6,7,8$, and 10 and the unit-cell lenght $L_{\text {cell }}$ increases as $19.3 a_{0}, 22.5 a_{0}, 25.7 a_{0}$, and $32.1 a_{0}$, respectively. The unit cell with eight electrons corresponds to a magic spherical cluster and that of ten electrons corresponds to the pair of magic clusters of eight and two electrons. The critical values for the unstable radii of $R$ $=5.5 a_{0}, 8.6 a_{0}, 11.6 a_{0}$, and $19 a_{0}$ are $L_{\text {cell }} / R=4.1,3.2,4.2$, and 4.8 , respectively; i.e., we obtain values near the classical value of 4.5. At the unstable radius of $R=15.5 a_{0}$, the wire is not deformed at least up to $L_{\text {cell }} / R=10$ (the largest length we have calculated), probably due to the fact that this radius lies in a beat of the supershell structure and it is actually relatively stable. We start all the calculations with a converged uniform potential profile along the wire axis (see Sec. III A). In this way we do not "add any energy" to the system when initiating the calculation. Therefore, if the wire starts to deform in the iteration process the reason is the disappearance of the local energy minimum. 

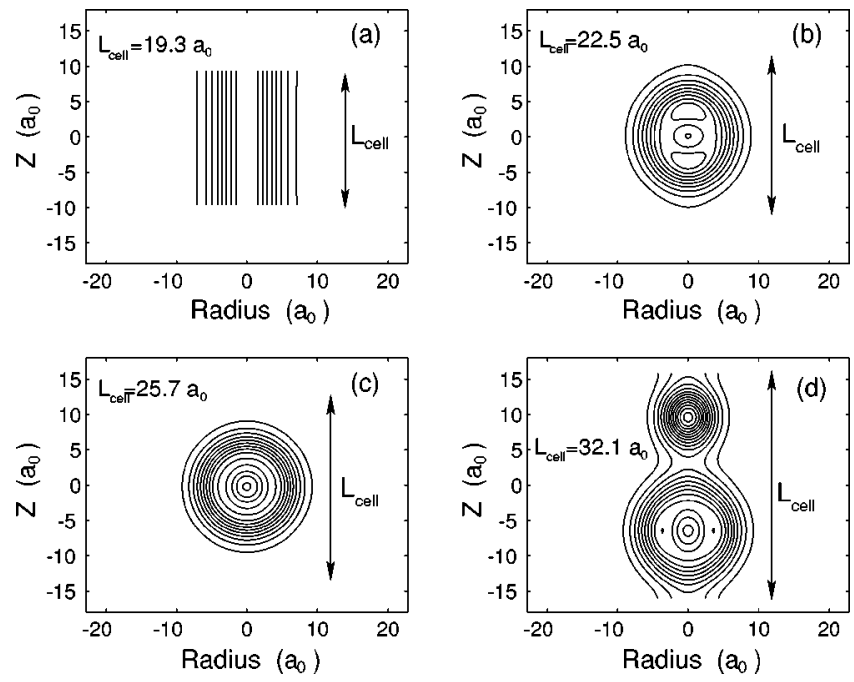

FIG. 2. Periodic infinite UJ wire with the nominal radius of $R$ $=5.5 a_{0}$ and $6(\mathrm{a}), 7(\mathrm{~b}), 8(\mathrm{c})$, and $10(\mathrm{~d})$ electrons in the periodic unit cell. The figures show the electron density of one unit cell. The contour spacing is 0.15 times the UJ bulk density value.

In addition, we narrow a stable uniform wire by increasing the length $L_{\text {cell }}$ of the periodic cell and maintaining the number of electrons constant. Each elongation step is solved self-consistently until convergence is reached. We observe that during the first steps the wire remains uniform, but at some point, before breaking into isolated clusters, the wire spontaneously deforms. Thus, we confirm self-consistently and dynamically the hypothesis by Kassubek et al. ${ }^{43}$

\section{Breaking of supported finite nanowires}

In order to study the formation and evolution of nanoconstrictions between two supporting leads, we follow the next procedure. First, we fix the number of electrons in the periodic supercell and solve self-consistently for the electronic structure of a uniform UJ wire having a stable magic radius. Then, the potential at both ends of the periodic cell is "frozen." This means that, although the Kohn-Sham equations are solved in the whole wire, in these regions the potential is not updated in the self-consistency process. The function of this "frozen" part is to emulate the lead parts where ion rearrangement does not occur as efficiently as at the constriction. In our calculation, these leads serve as handles to grab the UJ and pull it. The rest of the wire, the UJ at the middle part of the supercell, is the place where the wire will stretch. A sketch of the configuration is shown in Fig. 3. A sharp change in the potential between the constriction and the leads turned out to cause difficulties in numerical calculations. Therefore, we smooth out the potential at the left edge using the form

$$
F\left(z-z_{\text {edge }}^{1}\right) V_{\text {frozen }}+F\left(z_{\text {edge }}^{1}-z\right) V_{\mathrm{UJ}}
$$

where $F$ is a Fermi function with half-width of $0.5 a_{0}, V_{\text {frozen }}$ is the "frozen" potential, and $V_{\mathrm{UJ}}$ is the self-consistent UJ potential. For the right edge an analogous mixing is used. The main properties of the nanowire will not depend on the

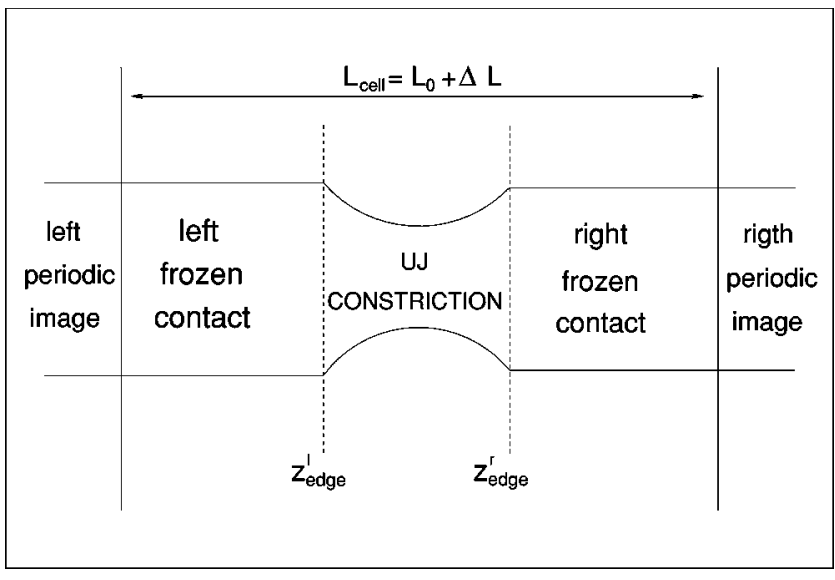

FIG. 3. Schematic view of the model system for simulations of breaking of finite nanowires supported by two leads.

particular choice of this matching because the physical features are determined by the narrowest part of the constriction.

We perform simulations starting with radii between $7.3 a_{0}$ and $20.7 a_{0}$, and changing the number of electrons initially in the constriction. The elongation of the wire is made in steps of about $1 a_{0}$, and always starting from the previous converged density, so that the grid spacing of the point mesh is increased to enlarge the cell. In order to overcome the interactions between the constriction and its periodic replica, ${ }^{44}$ we choose the length of the lead part to be 6 or more Fermi wavelengths $\left(\lambda_{F}=13.7 a_{0}\right)$. Throughout the rest of the paper we will use $\Delta L$ for the elongation; $\Delta L=0$ for the first step.

In Fig. 4, we show snapshots of the electronic density for a wire with the starting radius of $10.7 a_{0}$. The UJ part corre-

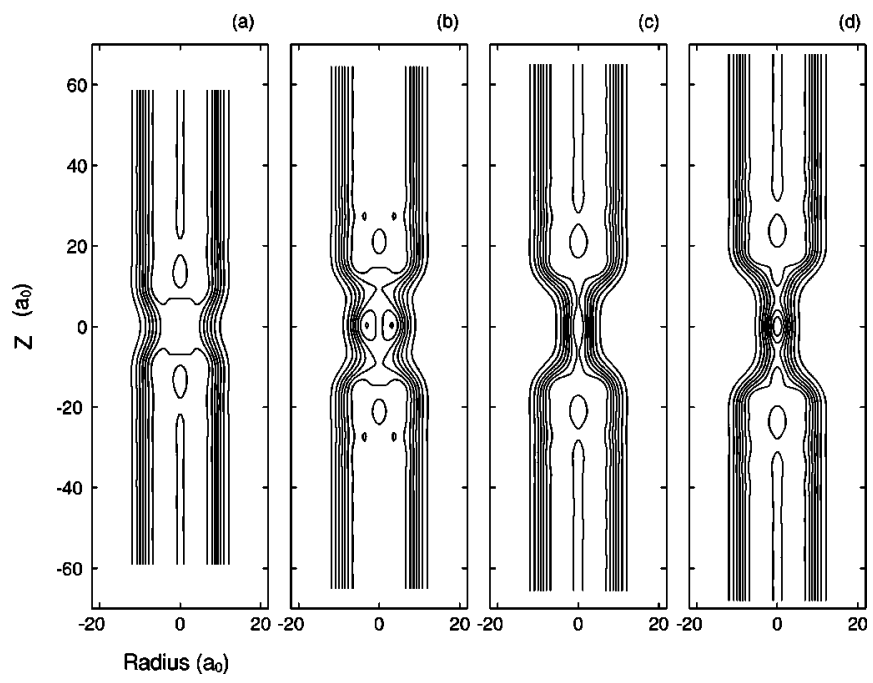

FIG. 4. Supported UJ wire. The UJ constriction contains eight electrons. Density contour plots for four different elongation lengths: $\Delta L=7.9 a_{0}$ (a), 19.8 $a_{0}$ (b), 20.8 $a_{0}$ (c), and $25.8 a_{0}$ (d) are shown. The snapshots in (b) and (c) are from consecutive selfconsistent calculations and the snapshot (d) is the last step before the nanowire breaking. The contour spacing is 0.15 times the mean UJ bulk density value. 
sponds to eight UJ electrons placed initially in the neck region. Electrons are free to move inside or outside the leads, depending on the requirements of the self-consistent solution. However, there are always about eight electrons in the constriction. Although this is one of the smallest wires we have calculated, it shows all the main features observed when simulating also larger wires.

If the breaking of an UJ nanowire would happen as for fluid between the leads, the electron density should evolve forming a catenoid-shaped surface. Similar shapes (such as hyperbolic, ${ }^{45}$ parabolic, ${ }^{23}$ cosine, ${ }^{46}$ etc.) have been used before to model the nanoconstriction in simple free-electron or jellium simulations. The main results, when the comparison is possible, have been essentially the same irrespective of the actual shape. In Fig. 4(a), the electron density is shown after the elongation of $\Delta L=7.9 a_{0}$. The catenoidlike density profile appears as expected for a classical fluid. When we continue elongating the nanowire the shape of the electron density changes dramatically from the classical one. If the distance between the leads is short, the electrons are strongly trapped at the narrowest part and they do not have much freedom in the rearrangement process. When the length of the constriction is large enough, the electrons have more space and freedom to achieve different types of energetically preferred shapes. In Fig. 4 (b), $\Delta L=19.8 a_{0}$ and the electrons in the constriction form a CDS. The electron density per unit length has two minima at both sides of the CDS and there are 7.1 electrons between these narrowest cross sections. The embedded cluster reminds the closed-shell cluster of eight electrons, but there are some differences. There are not enough electrons and the symmetry is not exactly spherical. It seems that the $p_{z}$ orbital ( $z$ along the cylinder axis) of the cluster has disappeared. We will analyze the structure in more detail below. Figure 4(c) shows the next consecutive elongation step with $\Delta L=20.8 a_{0}$. Note that the CDS disappears and a sudden change in the mean radius happens. In fact, the conductance changes simultaneously abruptly from $3 G_{0}$ to $1 G_{0}$ [see the inset in Fig. 5(a)]. At this point it is also remarkable that the shape of the constriction is again far from the catenoid having a constant magic radius. Figure 4(d) is for $\Delta L=25.8 a_{0}$, the last step before the nanowire breaks. Again a CDS appears during the elongation from the third to the fourth snapshot. There are 1.8 electrons between the two minimum cross sections at both sides of the CDS. This CDS can be interpreted as an embedded two-electron cluster. We observe that the radius of the constrictions is more or less constant with the same value as in the previous snapshot in Fig. 4(c).

At this point we want to focus on one characteristic property of UJ found when simulating the wire breaking: the UJ matter deforms very easily. This ability to deform allows the formation of the cylinders of magic radii glued to the leads. The radius jumps from one magic radius to the next through an abrupt charge reorganization. The CDS's of about two or eight electrons appear before the last charge reorganizations and the wire breaking. If there is enough $\mathrm{UJ}$ between the leads, suspended long thin cylinders appear and in the last steps they alternate with chains of CDS's producing a very extended elongation process. Here we want to underline that

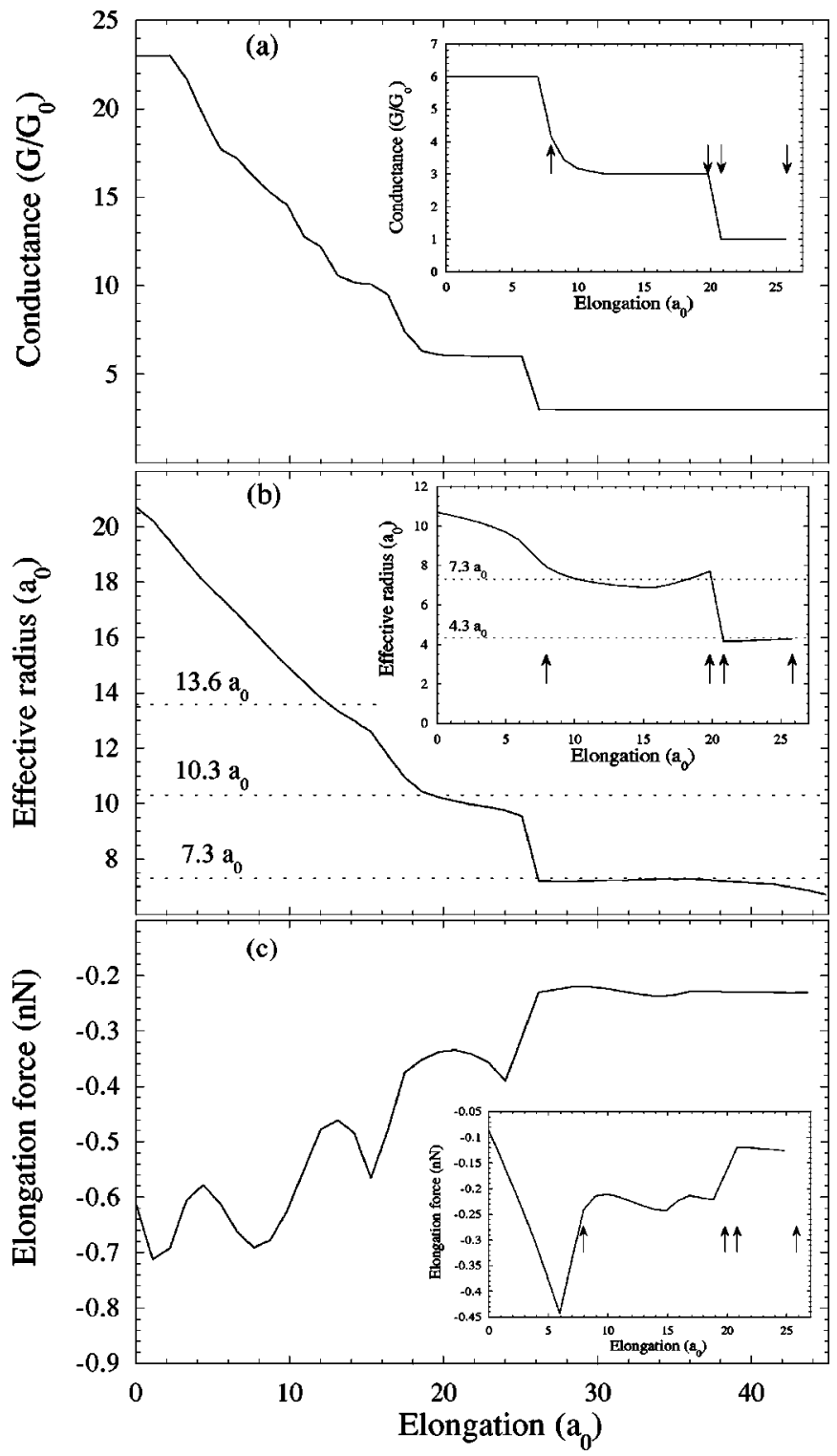

FIG. 5. Main figures: conductance, effective radius at the constriction, and elongation force for a wire with initial radius $R$ $=20.7 a_{0}$ and about $60 \mathrm{UJ}$ electrons in the constriction. Insets: the same quantities for the wire in Fig. 4 with initial radius $R=10 a_{0}$ and eight UJ electrons in the constriction. The arrows mark the points where the density has been plotted in Fig. 4 .

the CDS formation is a process different from the stability of a uniform cylindrical wire against the formation of a chain of spheres studied in Sec. III B. In that section, the quantummechanical shell structure may conserve the cylindrical structure that is not classically stable, whereas now the quantum-mechanical shell-structure effect destroys the classical catenoid type of solution producing a CDS in the constriction.

In Fig. 5, we show the conductance, the effective radius, and the elongation force as a function of the elongation for two different wires. The main figures correspond to an initial configuration with the radius of $20.7 a_{0}$ and 60 electrons in the UJ constriction. The insets display the results for a wire with an initial radius of $10.7 a_{0}$ and eight electrons in the 
(a)

(b)

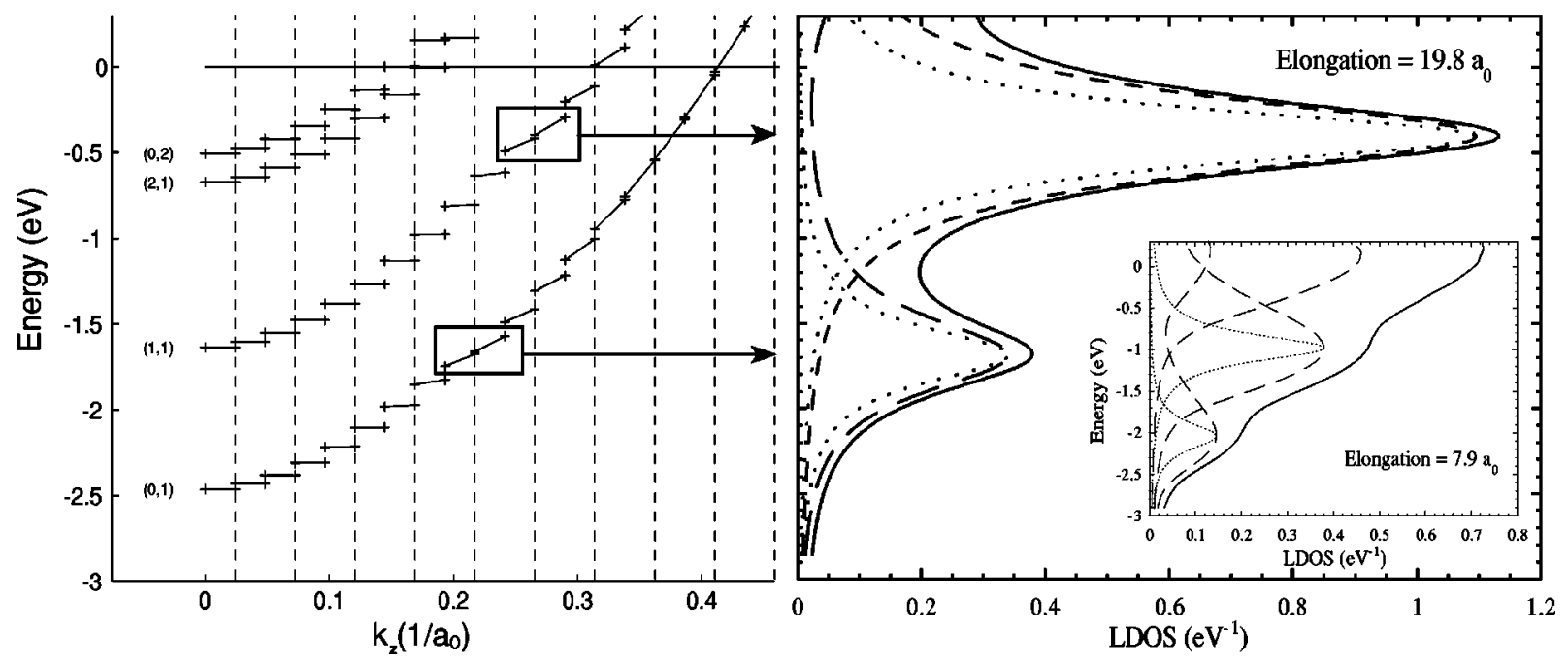

FIG. 6. (a) Electron band structure of the wire having eight UJ electrons and the elongation of $\Delta L=19.8 a_{0}$ [Fig. 4(b)]. The difference between the vertical dashed lines is one reciprocal lattice vector $\left(2 \pi / L_{\text {cell }}\right)$. The label for each branch represent the $(m, n)$ subband for the infinite wire. The energy eigenvalues are solved at two $k_{z}$ points: at the origin and at the zone boundary of the supercell Brillouin zone. (b) LDOS integrated between the two narrowest points of the electron density in Fig. 4(b) is displayed. The solid line represents the total ILDOS, the dashed lines are the contributions to the ILDOS of the states with $m=0$ (long-dashed line) and $m=1$ (short-dashed line). The dotted lines show the DOS of hypothetical localized states. The states marked with squares in the band structure are the main states contributing to the ILDOS. In the inset, the analogous plot for the ILDOS in the constriction of Fig. 4(a) with $\Delta L=7.9 a_{0}$ is shown. The origin of energy is the Fermi level.

constriction. The electron density of the latter wire is plotted in Fig. 4 at certain elongation stages.

The conductance is calculated with the adiabatic and semiclassical approximation used by Brandbyge et al. ${ }^{3}$ The constriction is divided into transversal slices. Then for each slice a uniform wire with the radial extent of the slice is built and the energy eigenvalues of the subband bottoms are calculated for this slice. The subband bottoms give effective potentials along the wire axis. If we look at the dependence of one of them on the position, we see that it raises at the constriction due to the strong confinement (see Fig. 8). The electrons in this subband at the Fermi energy of the leads have to overcome this barrier in order to carry current. To evaluate the transmission probability of the electrons at the Fermi level through the barrier, the semiclassical WKB formula is used.

The properties of the nanowires have been demonstrated to be dominated by the narrowest part of the constriction. Therefore we calculate an effective radius by evaluating the electron density per unit length at the middle of the wire. It is obtained with the value of the bulk electron density (corresponding to $r_{s}=4.176 a_{0}$ ). Figure 5(b) shows the effective radius as a function of the elongation of the wire. The plateaus or shoulders are in good coincidence with the infinite wire magic radii of $10.3 a_{0}, 7.3 a_{0}$, and $4.3 a_{0}$. For the larger wire shown in Fig. 5(b) also a small kink can be seen at $\Delta L=15.5 a_{0}$, which corresponds to the magic radius of $13.6 a_{0}$. Wider magic radii do not appear because of the beat region of the supercell structure. In the inset at the end of the plateaus the effective radius increases when elongating the wire due to the CDS formation. The sudden decrease of the effective radius, accompanied by a step in the conductance, is due to the sharp charge rearrangements in the constriction.

The elongation force, shown in Fig. 5(c), is evaluated as the negative derivative of the total energy with respect to the elongation. The rearrangement of the wire charge leads to discontinuous upward steps in the force, while if the radius changes smoothly the force draws a continuous buckling curve. At this point we want to point out the superiority of the UJ model in the force calculation over other jellium models. $^{22-24}$ In contrast with the experiments, ${ }^{1,2}$ the latter show a continuous behavior of the force without any steps. Moreover, for narrow constrictions positive values are obtained when the wire crosses an unstable zone. Note that in our model the force is always negative, as observed in the experiments ${ }^{1,2}$ and in atomistic simulations. ${ }^{3,13,14,47}$ Figure 5 shows clearly that the transport, geometrical, and mechanical properties of the nanowires under elongation are related.

\section{Electronic cluster-derived structures}

Let us now analyze more closely the CDS appearing in Fig. 4(b). In order to enlighten the origin of this structure, we plot in Fig. 6(a) the single-particle energy spectrum of the wire. The extended zone scheme is used for clarity. The labels on the left of each branch represent the corresponding $(|m|, n)$ subbands for the infinite wire. In practice, $n$ is obtained by calculating the number of radial nodes at the cell boundaries (see Fig. 3). The branches have the characteristic parabolic shapes, but they show two different stages. In the lower part of the parabolic subbands the eigenvalues form flat plateaus without $k_{z}$ dispersion. These states correspond 

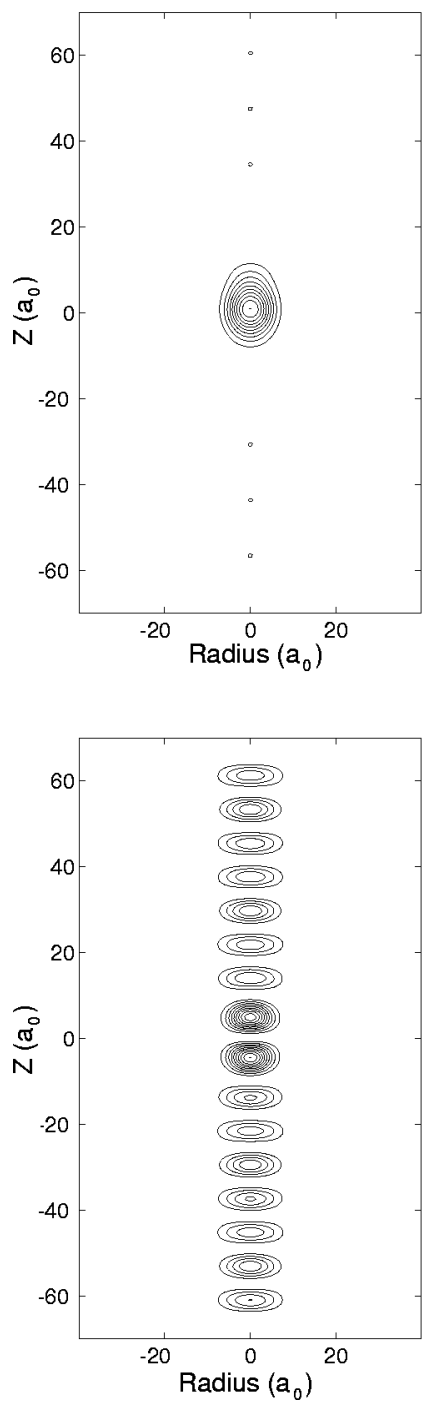

(a)

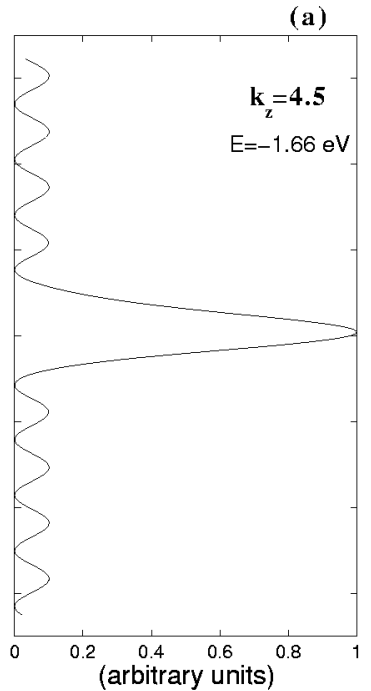

(c)

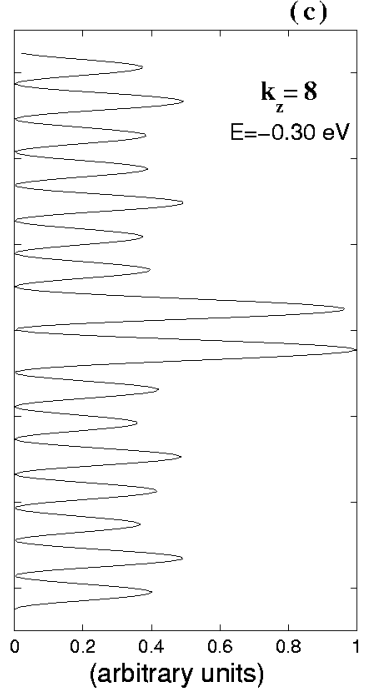

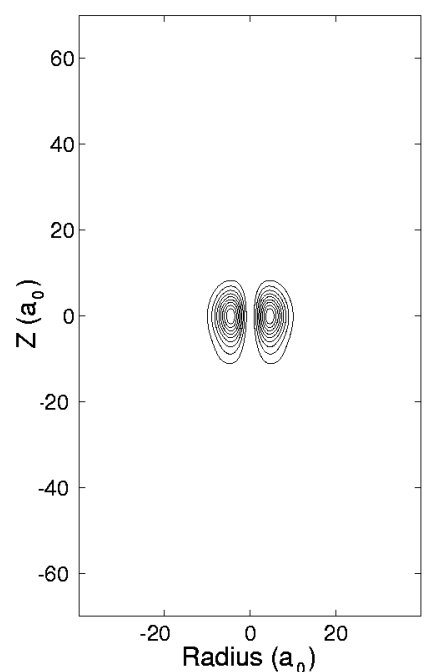

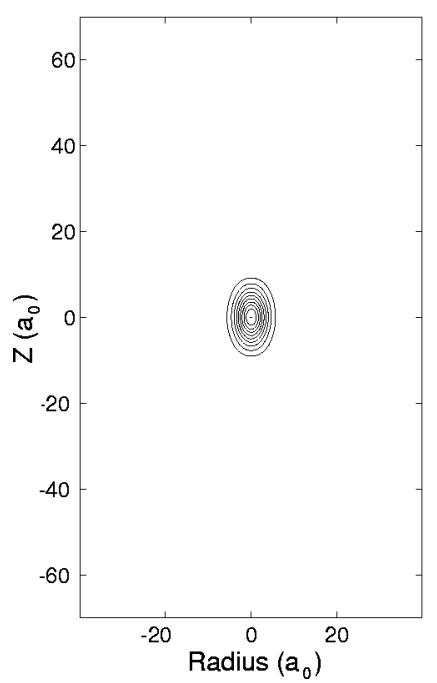

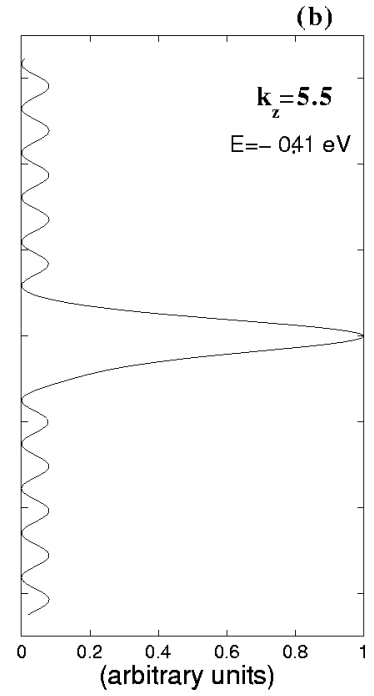

(b)

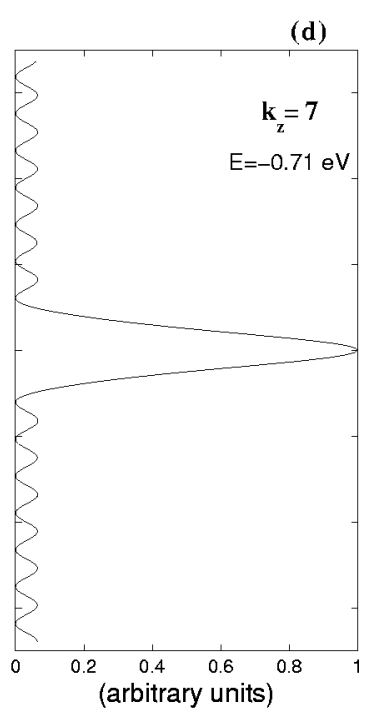

FIG. 7. (a), (b), and (c): Selected single-electron states in the wire having eight UJ electrons and the elongation of $\Delta L=19.8 a_{0}$ [Figs. 4(b) and 6]. Contour and profile plots (along the wire through the maximum value) of the squared moduli of the wavefunctions are shown. Plot (a) corresponds to a $m=0$ resonance state at the low-energy peak in the ILDOS (Fig. 6). Plot (b) corresponds to a $m=1$ resonance state at high energy. Plot (c) is an extended state of the $m=0$ subband at the energy of the $m=1$ peak in the ILDOS. Plot (d) is a localized state with $m=0$ corresponding to the elongation of $\Delta L=25.8 a_{0}$ [Fig. 4(c)]. The contour spacing is one-tenth of the maximum value. The $k_{z}$ vector is given in reciprocal-lattice vector units $\left(2 \pi / L_{\text {cell }}\right)$.

to the wave functions localized at the leads and they vanish at the center of the constriction. Therefore the $(0,2)$ and $(2,1)$ subbands cannot carry current through the constriction and they are closed channels. On the other hand, the states of the upper part of the $(0,1)$ and $(1,1)$ branches are extended along the whole wire and they form a continuous band (with the exception of small band gaps). The conductance of the wire is thus $3 G_{0}$ due to the extended states of the $(0,1)$ and $(1,1)$ open channels at Fermi energy. This conclusion is in accordance with the value obtained with the WKB approach.

In Fig. 6(b), we plot the integrated local density of states (ILDOS) in the constriction for the band structure of Fig. 6(a). It is calculated by integrating the local density of states (LDOS) over the space between the two narrowest parts in the electron density in Fig. 4(b). The LDOS itself is obtained by substituting the discrete energy levels with Lorenzians of full width at half maximum of $0.4 \mathrm{eV}$ and weighting them by the local probability amplitudes of the states in question. The ILDOS has two clear peaks, and while decomposing it we can see that the lower and the higher peak have the $m=0$ and $m=1$ character, respectively. The contribution of the $m$ $=2$ states is negligible. The two ILDOS peaks can be fitted by two energy levels convoluted with the same Lorenzian as the eigenlevels in the LDOS calculation. The resulting resonance peaks are shown in Fig. 6(b) by dotted lines. The positions and the heights of these peaks have been fitted manually. The coincidence between the fit and the true ILDOS is remarkable. In the inset of Fig. 6(b), we plot the LDOS integrated between the leads for the electron density showed in Fig. 4(a) having no CDS. We observe that the ILDOS is much smoother and it is similar to the DOS of an infinite wire with delocalized states. The different $m$ contri- 


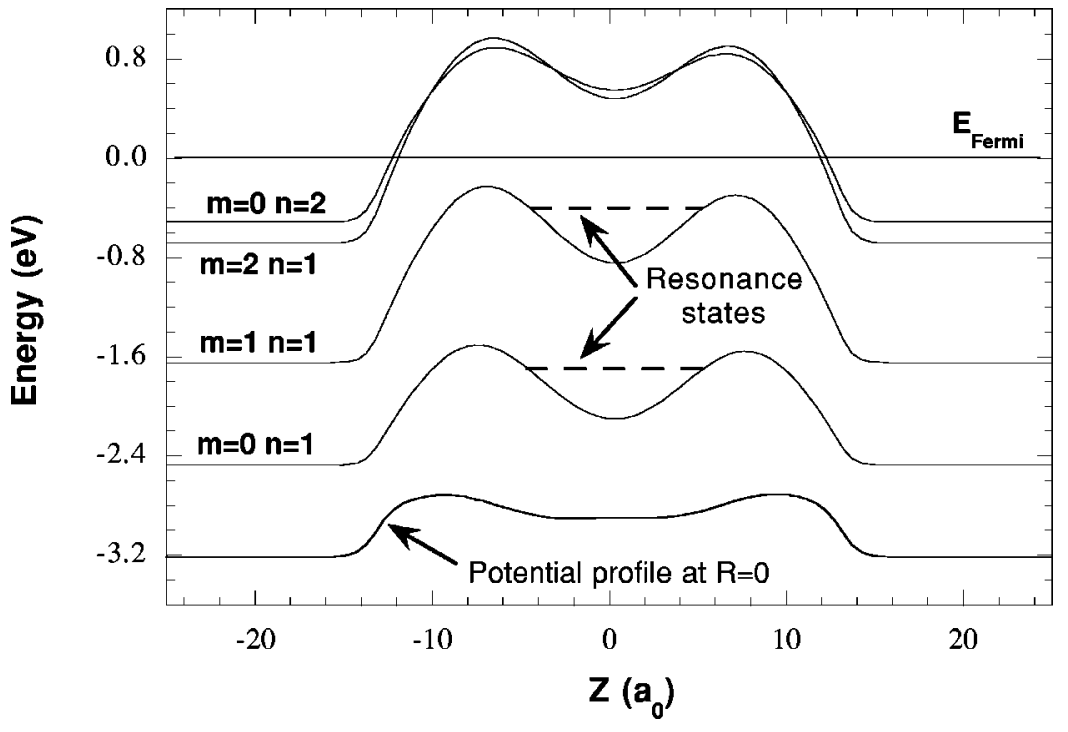

FIG. 8. Effective $z$-dependent potentials for the different $(m, n)$ channels. The wire has eight UJ electrons and the elongation of $\Delta L=19.8 a_{0}$ [Figs. 4(b) and 6]. The dashed lines in the potential wells are drawn at the energies of the resonance states. The lowest line is the bare KohnSham potential at $R=0$. butions cannot be fitted by single resonance peaks as shown by the dotted peak for the $m=0$ and $m=1$ contributions. Moreover, the inset shows that the $m$-decomposed peaks are slightly asymmetric with a tail on the high-energy side. These tails, which are not observable in the main figure in which the CDS appears, are due to the $\sqrt{\epsilon}$ dependence of the subband peaks in the DOS for infinite wires.

The ILDOS analysis suggests that in the energy subbands or branches, at the transition points from states localized in the leads to states extended across the whole wire [see Fig. 6(a)], rather localized resonance states appear in the constriction. To clarify this point, we plot selected states at the ILDOS peak energies in Fig. 7. Figures. 7(a) and 7(b) show clearly the localized character of the wave functions in the constriction at these energies. The state in Fig. 7(a) can be identified as the $1 s$ orbital of an eight-electron cluster. The second well-localized state [Fig. 7(b)], has $m=1$. Therefore it is doubly degenerate and it is identified as the $p_{x y}$ orbital. At about the energy of this $p_{x y}$ orbital, a $p_{z}$ orbital (directed along the wire axis) should appear in the $m=0$ branch in order to complete the eight-electron cluster. However, we do not find such a state with a strong localization in the constriction. As shown in Fig. 7(c), the $p_{z}$-type states are much more delocalized than the $p_{x y}$ resonance states. The difference reflects the fact that due to the orientation the interaction of the cluster $p_{z}$ orbital with the lead states is much stronger than that of the $p_{x y}$ orbital. The absence of a welllocalized $p_{z}$ orbital explains the clearly nonspherical shape of the embedded cluster in the electron density plot of Fig. 4(b), and also the finding that there are only 7.1 electrons in the constriction between the two narrowest cross sections.

Figure $7(d)$ shows a well-localized state for the wire with eight UJ electrons at the elongation of $\Delta L=25.8 a_{0}$ [Fig. $4(d)]$. The state can be identified as the $1 s$ orbital of a twoelectron cluster glued to the leads. There are 1.8 electrons between the two narrowest cross sections of the constriction supporting the assumption that this state is related to a twoelectron cluster.

The states in Fig. 7 have always a wavy, nondecaying background. This is a characteristic of resonance states; truly localized states would decay exponentially. The wavy background corresponds to the wave function of the leads (plane wave) at the energy that matches with that of the cluster state. To check this assumption we realize that the wavelength of the plane-wave background corresponds to the $k_{z}$ quantum number in the extended zone scheme. There are indeed two maxima in the modulus of the wave function per every Brillouin-zone unit of $k_{z}$ (see the labels of each wave function).

The existence of resonance states is related to the shape of the self-consistent potential having a small potential well in the nanoconstriction. To point out how this potential can admit a resonance state, we show in Fig. 8 the effective potential for states with different $(m, n)$ quantum numbers, calculated within the adiabatic approximation for the wire with eight UJ electrons and the elongation of $\Delta L=19.8 a_{0}$ [Fig. 4(b)]. We see that electrons at the constriction feel the existence of a potential well. We plot the energies corresponding to the ILDOS peaks with dashed lines and note that they lie exactly in the potential wells, where the resonances situate. It is also evident that an occupied resonant $p_{z}$ state does not occur because its energy eigenvalue should be well above the effective potential of the $(0,1)$ branch and because the potential well of the $(0,2)$ branch is above the Fermi level. In addition, by the help of Fig. 8 we can explain the different parts of the electron energy bands in Fig. 6(a). The states with energies above the effective potential maxima are extended along the whole wire. These are the current-carrying states of each branch. The states below the potential minimum of the constriction are trapped in the leads, corresponding to flat plateaus in the lower part of the energy branches [Fig. 6(a)]. Finally, between the potential maxima and the local minimum in the center we find resonant states that are enhanced at the constriction although they continue as plane waves in the leads.

\section{CONCLUSIONS}

We studied the stability of nanowires and the nanowire breaking process performing self-consistent calculations 
within the ultimate jellium model. In the model, electrons and positive background charge acquire the optimal density minimizing the total energy. The model enables thus studies of shape-dependent properties of nanoscopic systems such as quantum dots or, as in the present work, quantum wires. The model advocates the idea that the electronic structure determines, via the shell structure, the geometry and ionic structure also in a partially confined system.

First, we analyzed the stability of infinite periodic quantum wires pointing out the ability of the electronic band structure to stabilize the nanowires at magic radii, i.e., any small deformation of the nanowire along the $z$ axis always increases the energy. At the unstable radii corresponding to maximum values of the energy oscillations, the wire is uniform up to a critical value of the unit-cell length. The critical values found are close to the classical value of $L_{\text {cell }} / R$ $=4.5$. Above this limit the local energy minimum disappears and a deformation of the wire lowers the total energy.

Then we investigated the elongation process of finite nanowires supported by leads. The elongation force, conductance, and effective radius of the constriction were calculated simultaneously. The importance of the charge relaxation, in order to obtain results in agreement with the experiments, was shown, e.g., in the case of the elongation force. The ability of the ultimate jellium (electron density) to acquire the optimal shape allows the formation of CDS's showing the importance of electron states in the formation of these structures. The related resonance states and their origin was also shown. We found CDS's that can be linked with the eight- and two-electron free-standing clusters.

\section{ACKNOWLEDGMENTS}

One of the authors (E.O.) acknowledges the Spanish Ministerio de Ciencia y Tecnología for financial support under the Project No. PB98-0870-C02, and the Laboratory of Physics of the HUT for the kind hospitality. T.T. acknowledges financial support from the Vilho, Yrjö, and Kalle Väisälä foundation. This work has also been supported by the Academy of Finland through its Center of Excellence Program (2000-2005).
*Electronic address: eoa@we.lc.ehu.es

${ }^{1}$ G. Rubio, N. Agrait, and S. Vieira, Phys. Rev. Lett. 76, 2302 (1996).

${ }^{2}$ G. Rubio-Bollinger, S. Bahn, N. Agrait, K. Jacobsen, and S. Vieira, Phys. Rev. Lett. 87, 026101 (2001).

${ }^{3}$ M. Brandbyge, J. Schiøtz, P. Stoltze, K. Jacobsen, and J. Nørskov, Phys. Rev. B 52, 8499 (1995).

${ }^{4}$ A. Yanson, I. Yanson, and J. van Ruitenbeek, Phys. Rev. Lett. 84, 5832 (2000).

${ }^{5}$ A. Yanson, I. Yanson, and J. van Ruitenbeek, Nature (London) 400, 144 (1999).

${ }^{6}$ A. Yanson, I. Yanson, and J. van Ruitenbeek, Phys. Rev. Lett. 87, 216805 (2001).

${ }^{7}$ M. Brack, Rev. Mod. Phys. 65, 677 (1993).

${ }^{8}$ W. de Heer, Rev. Mod. Phys. 65, 611 (1993).

${ }^{9}$ V. Rodrigues, T. Fuhrer, and D. Ugarte, Phys. Rev. Lett. 85, 4124 (2000).

${ }^{10}$ V. Rodrigues, J. Bettini, A. Rocha, L. Rego, and D. Ugarte, Phys. Rev. B 65, 153402 (2002).

${ }^{11}$ H. Ohnishi, Y. Kondo, and K. Takayanagi, Nature (London) 395, 780 (1998).

${ }^{12}$ A. Yanson, G.R. Bollinger, H. van den Brom, N. Agraï, and J. van Ruitenbeek, Nature (London) 395, 783 (1998).

${ }^{13}$ U. Landman, Solid State Commun. 107, 693 (1998).

${ }^{14}$ M. Sørensen, M. Brandbyge, and K. Jacobsen, Phys. Rev. B 57, 3283 (1998).

${ }^{15}$ H. Häkkinen and M. Manninen, Europhys. Lett. 44, 80 (1998).

${ }^{16}$ E. da Silva, A. da Silva, and A. Fazzio, Phys. Rev. Lett. 87, 256102 (2001).

${ }^{17}$ D. Sánchez-Portal, E. Artacho, J. Junquera, P. Ordejón, A. García, and J. Soler, Phys. Rev. Lett. 83, 3884 (1999).

${ }^{18}$ E. Tosatti, S. Prestipino, S. Kostlmeiner, A.D. Corso, and F.D. Tolla, Science 291, 288 (2001).

${ }^{19}$ R. Barnett and U. Landman, Nature (London) 387, 788 (1997).

${ }^{20}$ E. Ogando, N. Zabala, and M. Puska, Nanotechnology 13, 363 (2002).
${ }^{21}$ M. Puska, E. Ogando, and N. Zabala, Phys. Rev. B 64, 033401 (2001).

${ }^{22}$ N. Zabala, M. Puska, and R. Nieminen, Phys. Rev. B 59, 12652 (1999).

${ }^{23}$ C. Yannouleas, E. Bogachek, and U. Landman, Phys. Rev. B 57, 4872 (1998).

${ }^{24}$ C. Yannouleas and U. Landman, J. Phys. Chem. B 101, 5780 (1997).

${ }^{25}$ M. Manninen, Phys. Rev. B 34, 6886 (1986).

${ }^{26}$ M. Koskinen, P. Lipas, and M. Manninen, Z. Phys. D: At., Mol. Clusters 35, 285 (1995).

${ }^{27}$ M. Koskinen, P. Lipas, and M. Manninen, Europhys. Lett. 30, 519 (1995).

${ }^{28}$ J. Mandel and S. McCormick, J. Comput. Phys. 80, 442 (1989).

${ }^{29}$ M. Heiskanen, T. Torsti, M. Puska, and R. Nieminen, Phys. Rev. B 63, 245106 (2001).

${ }^{30}$ T. Torsti, M. Heiskanen, M. Puska, and R. Nieminen, Int. J. Quantum Chem. 91, 171 (2003); T. Torsti, M. Heiskanen, M. Puska, and R. Nieminen, cond-mat/0205056 (unpublished).

${ }^{31}$ R. Jones and O. Gunnarsson, Rev. Mod. Phys. 61, 689 (1989).

${ }^{32}$ J. Perdew and A. Zunger, Phys. Rev. B 23, 5048 (1981).

${ }^{33}$ D. Ceperley and B. Alder, Phys. Rev. Lett. 45, 566 (1980).

${ }^{34}$ H. Shore and J. Rose, Phys. Rev. B 59, 10485 (1999).

${ }^{35}$ J. Perdew, H. Tran, and E. Smith, Phys. Rev. B 42, 11627 (1990).

${ }^{36}$ H. Shore and J. Rose, Phys. Rev. Lett. 66, 2519 (1991).

${ }^{37}$ S. Reimann, M. Koskinen, J. Helgesson, P. Lindelof, and M. Manninen, Phys. Rev. B 58, 8111 (1998).

${ }^{38}$ S.F.M.A. Brandt and J.W. Ruge, SIAM J. Sci. Comput. (USA) 4, 244 (1983).

${ }^{39}$ A.C.D. Raczkowski and L.W. Wang, Phys. Rev. B 64, 121101(R) (2001).

${ }^{40}$ J.F. Annett, Comput. Mater. Sci. 4, 23 (1995).

${ }^{41}$ P. Ziesche, M. Puska, T. Korhonen, and R. Nieminen, J. Phys.: Condens. Matter 5, 9049 (1993). 
${ }^{42}$ J.P. Perdew, Y. Wang, and E. Engel, Phys. Rev. Lett. 66, 508 (1991).

${ }^{43}$ F. Kassubek, C. Stafford, H. Grabert, and R. Goldstein, Nonlinearity 14, 167 (2001).

${ }^{44}$ J.B.C.A. Stafford and D. Baeriswyl, Phys. Rev. B 84, 2548 (2000).
${ }^{45}$ J. Torres, J. Pascual, and J. Sáenz, Phys. Rev. B 49, 16581 (1994).

${ }^{46}$ C. Stafford, D. Baeriswyl, and J. Bürki, Phys. Rev. Lett. 79, 2863 (1997).

${ }^{47}$ A. Nakamura, M. Brandbyge, L. Hansen, and K. Jacobsen, Phys. Rev. Lett. 82, 1538 (1999). 